\title{
Inclisiran in Patients at High Cardiovascular Risk with Elevated LDL Cholesterol
}

\author{
Kausik K. Ray, M.D., UIf Landmesser, M.D., Lawrence A. Leiter, M.D., \\ David Kallend, M.D., Robert Dufour, M.D., Mahir Karakas, M.D., Tim Hall, M.D., \\ Roland P.T. Troquay, M.D., Traci Turner, M.D., Frank L.J. Visseren, M.D., \\ Peter Wijngaard, Ph.D., R. Scott Wright, M.D., \\ and John J.P. Kastelein, M.D., Ph.D.
}

The authors' affiliations are listed in the Appendix. Address reprint requests to Dr. Ray at the Imperial Centre for Cardiovascular Disease Prevention, Department of Primary Care and Public Health, Imperial College London, Reynolds Bldg., St. Dunstan's Rd., London W6 8RP, United Kingdom, or at k.ray@imperial.ac.uk.

Drs. Ray and Landmesser contributed equally to this article.

This article was published on March 17, 2017, at NEJM.org.

N Engl J Med 2017;376:1430-40. DOI: 10.1056/NEJMoal615758

Copyright (C) 2017 Massachusetts Medical Society.
A BSTRACT

\section{BACKGROUND}

In a previous study, a single injection of inclisiran, a chemically synthesized small interfering RNA designed to target PCSK9 messenger RNA, was found to produce sustained reductions in low-density lipoprotein (LDL) cholesterol levels over the course of 84 days in healthy volunteers.

\section{METHODS}

We conducted a phase 2, multicenter, double-blind, placebo-controlled, multipleascending-dose trial of inclisiran administered as a subcutaneous injection in patients at high risk for cardiovascular disease who had elevated LDL cholesterol levels. Patients were randomly assigned to receive a single dose of placebo or 200, 300 , or $500 \mathrm{mg}$ of inclisiran or two doses (at days 1 and 90) of placebo or 100, 200, or $300 \mathrm{mg}$ of inclisiran. The primary end point was the change from baseline in LDL cholesterol level at 180 days. Safety data were available through day 210, and data on LDL cholesterol and proprotein convertase subtilisin-kexin type 9 (PCSK9) levels were available through day 240 .

\section{RESULTS}

A total of 501 patients underwent randomization. Patients who received inclisiran had dose-dependent reductions in PCSK9 and LDL cholesterol levels. At day 180, the least-squares mean reductions in LDL cholesterol levels were 27.9 to $41.9 \%$ after a single dose of inclisiran and 35.5 to $52.6 \%$ after two doses $(\mathrm{P}<0.001$ for all comparisons vs. placebo). The two-dose 300 -mg inclisiran regimen produced the greatest reduction in LDL cholesterol levels: $48 \%$ of the patients who received the regimen had an LDL cholesterol level below $50 \mathrm{mg}$ per deciliter $(1.3 \mathrm{mmol}$ per liter) at day 180. At day 240, PCSK9 and LDL cholesterol levels remained significantly lower than at baseline in association with all inclisiran regimens. Serious adverse events occurred in $11 \%$ of the patients who received inclisiran and in $8 \%$ of the patients who received placebo. Injection-site reactions occurred in $5 \%$ of the patients who received injections of inclisiran.

\section{CONCLUSIONS}

In our trial, inclisiran was found to lower PCSK9 and LDL cholesterol levels among patients at high cardiovascular risk who had elevated LDL cholesterol levels. (Funded by the Medicines Company; ORION-1 ClinicalTrials.gov number, NCT02597127.) 
I OW-DENSITY LIPOPROTEIN (LDL) CHOLESterol is a causal factor in atherosclerotic carscular disease. Statins have been shown to reduce LDL cholesterol levels and cardiovascular events in large outcome trials, findings that have made them the therapeutic cornerstone of clinical practice. ${ }^{1}$ Despite the proven efficacy of statins, there is considerable variability in individual responses to these drugs. ${ }^{2}$ Furthermore, some observational data suggest that as many as half of persons who begin statin therapy discontinue it within a year. ${ }^{3}$ Moreover, among patients receiving statin therapy who are at high risk for cardiovascular disease and who have persistent elevation of LDL cholesterol levels, the rates of cardiovascular events remain high, necessitating the development of additional therapies.

The discovery that the serine protease proprotein convertase subtilisin-kexin type 9 (PCSK9) promotes the degradation of the LDL receptor affords an additional route through which LDL cholesterol levels in plasma can be controlled. ${ }^{4}$ Initial therapeutic approaches to reducing circulating levels of PCSK9 have focused on the use of monoclonal antibodies. This approach sequesters virtually all PCSK9 in the reticuloendothelial system, thus preventing it from binding to the LDL receptor. ${ }^{5,6}$ Circulating PCSK9 is derived almost entirely from the liver ${ }^{7}$; hence, therapeutic approaches that target hepatic production of PCSK9 may offer an alternative to the use of monoclonal antibodies.

RNA interference and related RNA silencing pathways provide an opportunity to harness a highly specific endogenous mechanism for regulating gene expression. ${ }^{8}$ Small interfering RNAs (siRNAs) selectively and catalytically silence the translation of their complementary target messenger RNAs (mRNAs) in a sequence-specific manner through the formation of effector RNAinduced silencing complexes. ${ }^{9,10}$ Inclisiran is an investigational, chemically synthesized siRNA molecule that has produced sustained hepatocytespecific, PCSK9-specific RNA silencing in healthy volunteers to 84 days after administration. ${ }^{11}$ Here, we present the results of the phase 2 ORION-1 trial, a dose-finding trial evaluating the efficacy of different inclisiran dosing regimens among patients who have elevated LDL cholesterol levels despite receiving the maximum possible dose of a statin and who are considered to be at high risk for atherosclerotic cardiovascular disease. Within the limits of the trial, we also evaluated the safety and efficacy of inclisiran in lowering LDL cholesterol levels.

\section{METHODS}

\section{TRIAL DESIGN AND OVERSIGHT}

ORION-1 was a randomized, double-blind, placebocontrolled, phase 2, multicenter trial. The objective was to evaluate the effects of different doses and dosing intervals for the use of inclisiran. The trial was designed by the principal investigator (the first author) and the sponsor, the Medicines Company, and was performed by the sponsor and World Wide Clinical Trials. Data were collected by the investigators, and data analyses were conducted by Statistics Collaborative and the Medicines Company. Ethics committee approval was obtained at all participating institutions. The first two authors wrote the first draft of the manuscript; editorial assistance was provided by Greensplash and funded by the Medicines Company. All the authors participated in revising the manuscript and vouch for the accuracy and completeness of the data and the fidelity of the trial to the protocol, which is available with the full text of this article at NEJM.org.

\section{PATIENTS}

Detailed inclusion and exclusion criteria are described in the Supplementary Appendix, available at NEJM.org. Men and women 18 years of age or older were eligible for participation in the trial if the LDL cholesterol level at screening was higher than $70 \mathrm{mg}$ per deciliter (1.8 mmol per liter) (for patients with a history of atherosclerotic cardiovascular disease) or higher than $100 \mathrm{mg}$ per deciliter (2.6 mmol per liter) (for patients without a history of atherosclerotic cardiovascular disease). Patients were required to have been receiving the maximum possible dose of a statin with or without additional lipid-lowering therapy at stable doses for at least 30 days before screening and were required to have no planned changes in background therapy during the course of the trial. Any use at any time of a monoclonal antibody drug targeting PCSK9 was an exclusion criterion and was prohibited during the trial. Written informed consent was obtained from all the patients before participation in the trial. 


\section{TRIAL PROCEDURES}

An interactive Web response system was used to randomly assign patients to one of eight study groups (Fig. S1 in the Supplementary Appendix); a single dose of placebo or 200,300 , or $500 \mathrm{mg}$ of inclisiran on day 1 or two doses of placebo or 100,200 , or $300 \mathrm{mg}$ of inclisiran on day 1 and day 90. Study-group assignments were stratified according to country and current use of statins or other lipid-modifying medications. End-of-trial evaluations were conducted at day 210 , with the exception of those for patients whose LDL cholesterol levels had not returned to within $20 \%$ of the starting level. For these patients, evaluations were conducted at day 210 and subsequently at follow-up visits every 30 days until day 360 or until the LDL cholesterol level returned to within $20 \%$ of the starting level (whichever came first).

\section{EFFICACY}

Fasting blood samples were obtained at each study visit, and LDL cholesterol levels were measured by combining ultracentrifugation with precipitation (beta quantification $)^{12}$ at Medpace laboratories that had been accredited by the National Heart, Lung, and Blood Institute Part III Lipid Standardization Program. A full description of the methods used to measure prespecified biomarkers is available in the Laboratory Analytical Methods subsection and Table S1 in the Supplementary Appendix.

\section{SAFETY}

Data on adverse events, vital signs, clinical laboratory values, and electrocardiograms were obtained at specified follow-up visits through the end-of-trial visit (day 210). Adverse events were classified as mild, moderate, or severe by the investigator with the use of standard Medical Dictionary for Regulatory Activities terms and according to system organ class. Injection-site reactions were an adverse event of special interest, and antidrug antibodies to inclisiran were evaluated.

\section{STATISTICAL ANALYSIS}

The primary efficacy end point was the percentage change from baseline in LDL cholesterol level at day 180 . Secondary efficacy end points included the percentage change in serum PCSK9 levels, lipid measures, and high-sensitivity C-reactive protein levels at day 180 and changes in these measures as well as in levels of LDL cholesterol at other time points.
A total of 480 randomly assigned patients were planned for inclusion in the trial: 60 patients in each of the six inclisiran treatment groups and in each of the two placebo groups (3:1 ratio of patients assigned to receive inclisiran or placebo). Under the assumption of a 15\% dropout rate, the sample required would be 50 patients who could be evaluated in each of the six inclisiran groups and approximately 100 patients who could be evaluated in total across the placebo groups (for a total of $\geq 400$ patients) to detect a 30 -percentagepoint difference in the percentage reduction from baseline in LDL cholesterol level between at least one inclisiran dose group and its corresponding placebo group with more than $90 \%$ power.

The primary end point was analyzed as the leastsquares mean percentage change from baseline to day 180 . This was calculated with a repeatedmeasurement linear-effects model, which included study group, baseline value, scheduled follow-up visit, and the interaction of study group with scheduled visit. The analysis was performed with the use of the PROC MIXED procedure in SAS software with an auto-regressive variance structure that incorporates treatment at each visit as fixed effects and patients as random effects. For both the primary and secondary end points, $\mathrm{P}$ values were adjusted for multiple comparisons with the use of Dunnett's test for comparison among the six inclisiran groups and the placebo comparator groups. Separate analyses were performed for each dosing strategy - that is, a single dose and two doses. The type I error significance level was 0.05 for a two-sided test.

All patients who received at least one dose of inclisiran or placebo were included in the safety analysis (safety population). The prespecified modified intention-to-treat population was defined as all randomly assigned patients who received at least one dose of study agent and for whom both the baseline and the 180-day follow-up LDL cholesterol level measurements were available. An intention-to-treat analysis was performed with the use of imputation, as described in the Supplementary Appendix, for patients with missing data.

Time-course data are presented as means with $95 \%$ confidence intervals. Variation in responses among patients is depicted graphically with waterfall plots. Analyses were performed with SAS software, versions 9.2 and higher (SAS Institute). Full details of the statistical analysis plan are available at NEJM.org. 


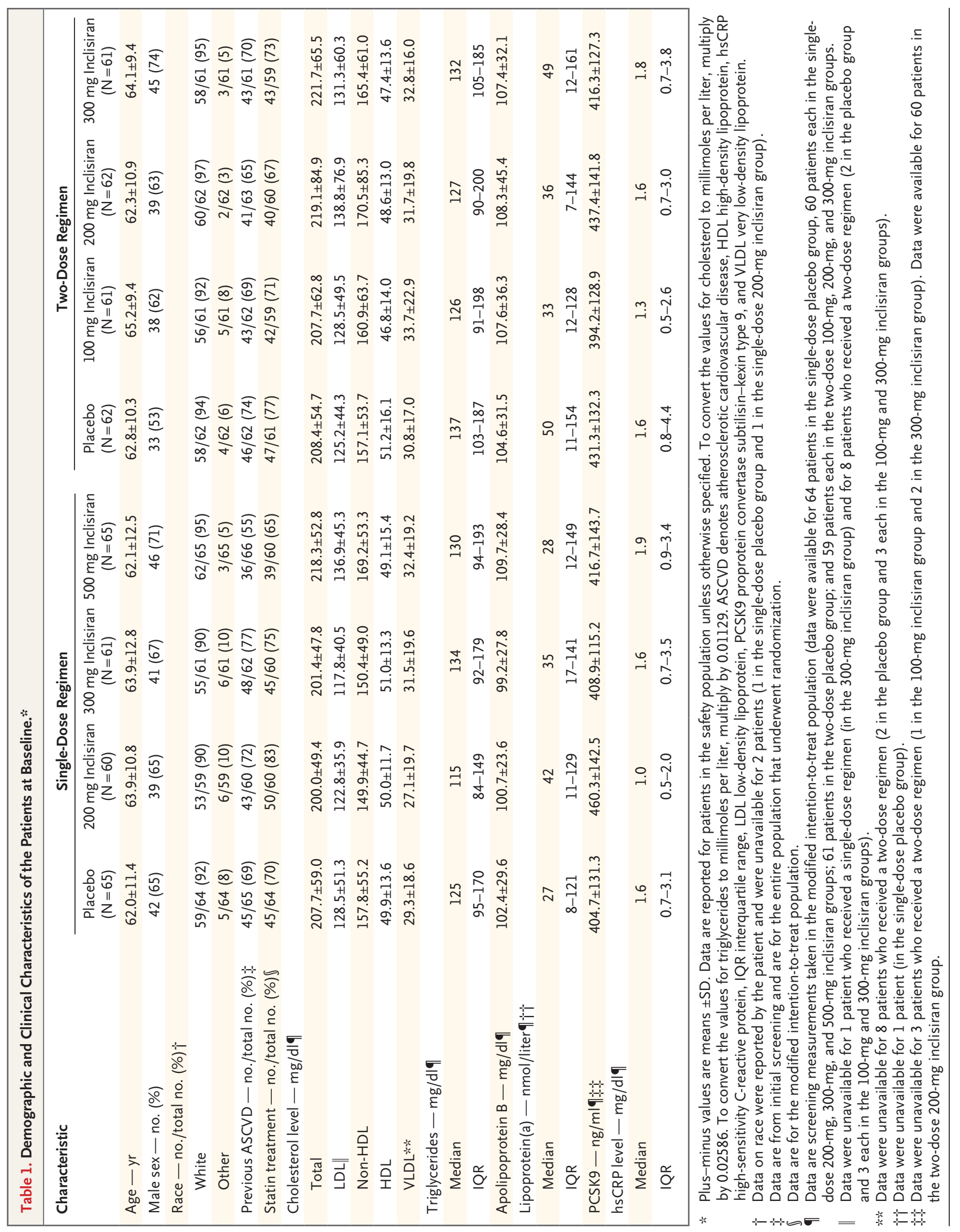




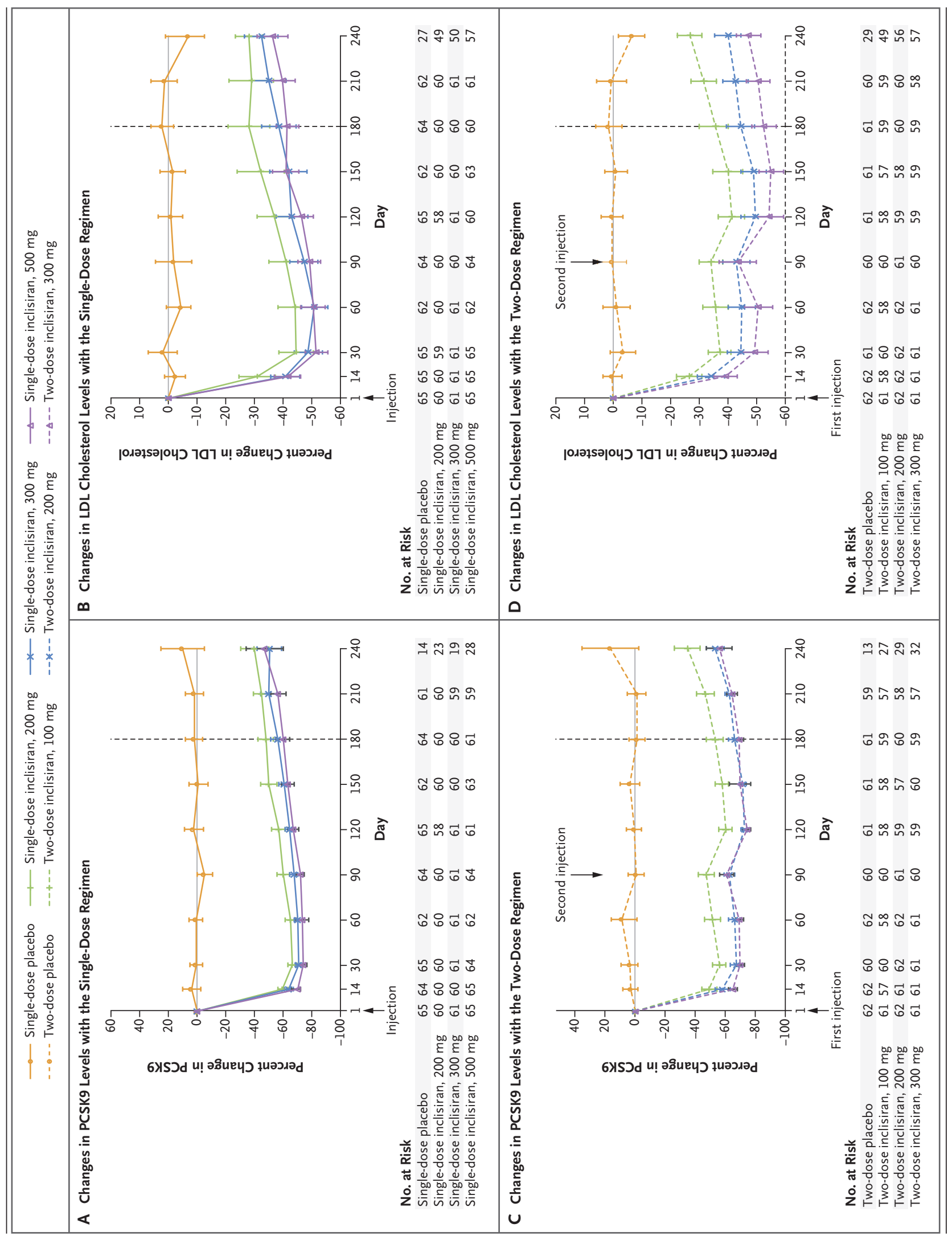


Figure 1 (facing page). Effect of Inclisiran on PCSK9 and Low-Density Lipoprotein (LDL) Cholesterol Levels. The data points are means, and the I bars indicate $95 \%$ confidence intervals. The dashed vertical line in each panel indicates day 180 , the day on which these end points were assessed.

\section{RESULTS}

\section{PATIENTS}

A total of 646 patients were screened, and 501 patients were randomly assigned to one of the two placebo groups or one of the six inclisiran groups (Figs. S1 and S2 in the Supplementary Appendix). Of these patients, 497 received inclisiran or placebo (1 patient each in the singledose $300 \mathrm{mg}$, single-dose $500 \mathrm{mg}$, double-dose $100 \mathrm{mg}$, and double-dose $200 \mathrm{mg}$ inclisiran groups did not receive an injection of inclisiran) and comprise the efficacy and safety populations. The baseline characteristics of the patients in each study group are provided in Table 1 and in Table S2 in the Supplementary Appendix. At study entry, $73 \%$ of the patients were receiving statin therapy, and $31 \%$ of the patients were receiving ezetimibe.

\section{PCSK9 LEVELS}

Fourteen days after a single injection of inclisiran, PCSK9 levels were reduced from baseline levels by a mean of 59.6 to $68.7 \%$ across the range of inclisiran doses from $100 \mathrm{mg}$ to $500 \mathrm{mg}$, whereas mean PCSK9 levels increased by $3.8 \%$ with placebo (Fig. 1A and 1C). At day 30, PCSK9 levels were reduced further, to between 66.2 and $74.0 \%$ below baseline levels, with similar reductions observed at day 60 and day 90. Among the patients who received a single-dose regimen, the mean reductions in PCSK9 levels at day 180 (a secondary end point) ranged between 47.9 and $59.3 \%$ ( $\mathrm{P}<0.001$ for each dose vs. placebo) (Table 2). In comparison, among the patients who received a two-dose regimen, further reductions in PCSK9 levels were observed after the second dose. At day 90, these patients had reductions of 47.0 to $62.8 \%$, and at day 120 , they had reductions of 60.4 to $74.5 \%$. At day 180, the mean reductions from baseline in PCSK9 levels among patients who received a two-dose regimen ranged between 53.2 and $69.1 \%$ ( $\mathrm{P}<0.001$ for each dose vs. placebo) (Table 2). In association with both the single-dose and two-dose inclisiran regimens, the reductions in PCSK9 levels at day 240 were greater than $40 \%$ (Fig. 1A and 1C).

\section{LDL CHOLESTEROL LEVELS AND OTHER LABORATORY MEASURES}

LDL cholesterol levels were already declining from baseline levels at 14 days after the first injection of inclisiran, and by day 30 , the mean reductions in LDL cholesterol level ranged between 44.5 and $50.5 \%$ below baseline across all inclisiran doses tested (Fig. 1B and 1D), with a nadir at approximately day 60 for the single-dose regimens and at day 150 for the two-dose regimens. The primary end point was the percentage reduction from baseline in LDL cholesterol level at day 180: the least-squares mean reductions were significantly greater after a single dose of inclisiran (27.9 to $41.9 \%$ reduction) than in association with placebo $(2.1 \%$ increase) $(\mathrm{P}<0.001$ for all inclisiran doses with regard to the primary efficacy end point) (Table 2). At day 240, the mean reductions in LDL cholesterol level from baseline ranged between 28.2 and $36.6 \%$ (Fig. 1B).

After the second dose of inclisiran, LDL cholesterol levels were decreased (with respect to levels at baseline) by 34.2 to $44.1 \%$ at day 90 and by 41.1 to $54.6 \%$ at day 120 . The differences between the two-dose regimens and placebo with regard to the primary end point were significant: at day 180 , the least-squares mean reductions in LDL cholesterol levels from baseline among patients who received a two-dose inclisiran regimen ranged from 35.5 to $52.6 \%$, whereas the placebo group had an increase from baseline of $1.8 \%$ ( $\mathrm{P}<0.001$ for all comparisons vs. placebo) (Table 2). Similar results were obtained with the use of imputation and an intention-to-treat analysis (Table S3 in the Supplementary Appendix). At day 240, the mean reductions in LDL cholesterol levels from baseline ranged between 26.7 and 47.2\% (Fig. 1D).

Among patients who received placebo against a background of the maximum possible dose of a statin, there was considerable variation at day 180 in the changes in LDL cholesterol levels from baseline (mean $[ \pm \mathrm{SD}]$ absolute difference, $-0.7 \pm 25.6 \mathrm{mg}$ per deciliter $[-0.02 \pm 0.66 \mathrm{mmol}$ per liter]) (Fig. 2A). In contrast, all patients who received two 300-mg doses of inclisiran had a decline in LDL cholesterol level at day 180 (mean absolute change in LDL cholesterol level, 


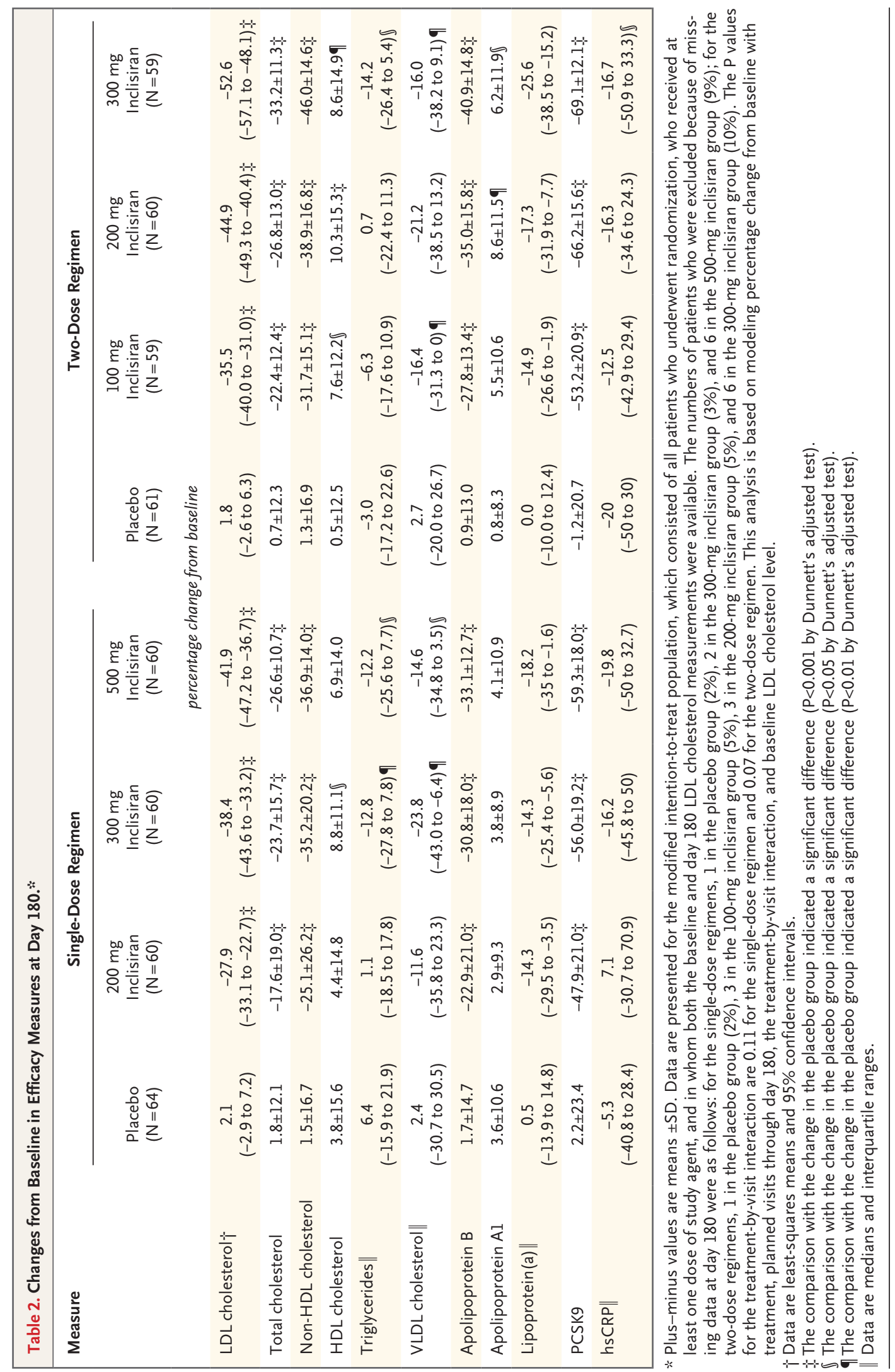




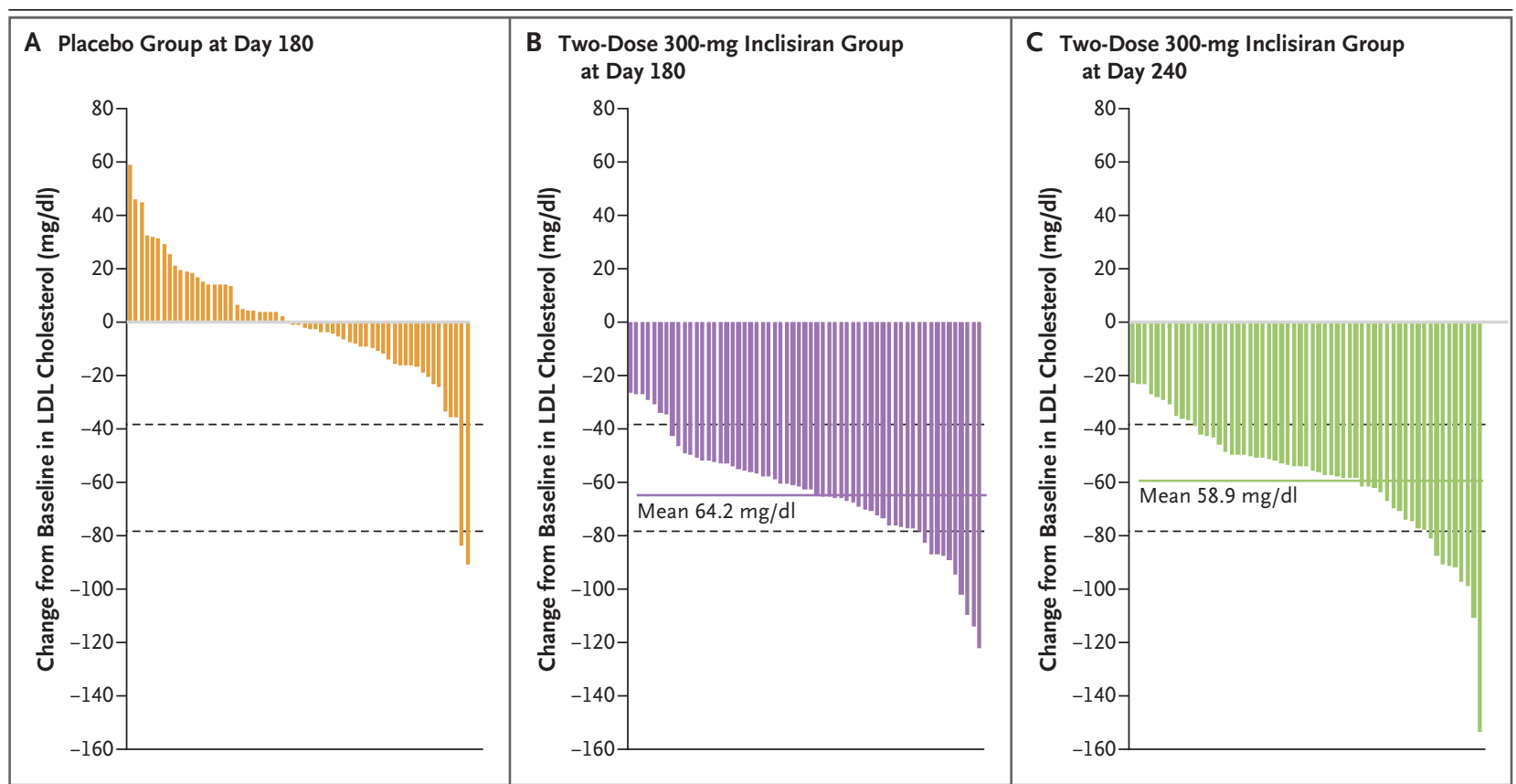

Figure 2. Changes in LDL Cholesterol Levels.

The change in LDL cholesterol level from baseline to day 180 is shown for each patient randomly assigned to the two-dose placebo group (61 patients) (Panel A) and the two-dose 300-mg inclisiran group (59 patients) (Panel B); the changes from baseline to day 240 are also shown for the two-dose 300-mg inclisiran group (59 patients) (Panel C). Dashed lines represent LDL cholesterol reductions of $39 \mathrm{mg}$ per deciliter and $78 \mathrm{mg}$ per deciliter. To convert the values for cholesterol to millimoles per liter, multiply by 0.02586 .

$-64.2 \pm 20.7 \mathrm{mg}$ per deciliter $[-1.66 \pm 0.54 \mathrm{mmol}$ per liter]) (Fig. 2B), and 54\% of the patients had a reduction of $50 \%$ or more (see the Supplementary Appendix). In this inclisiran dose group, 5\%, $48 \%$, and $66 \%$ of the patients had LDL cholesterol levels at day 180 of less than $25 \mathrm{mg}$ per deciliter $(0.65 \mathrm{mmol}$ per liter), less than $50 \mathrm{mg}$ per deciliter (1.3 mmol per liter), and less than $70 \mathrm{mg}$ per deciliter (1.8 mmol per liter), respectively. At day 240 , the individual cholesterol levels remained lower than at baseline in the same patient group (Fig. 2C).

We found significant reductions in levels of non-HDL cholesterol and apolipoprotein B and no significant change in levels of high-sensitivity C-reactive protein among patients randomly assigned to receive inclisiran. The percentage changes from baseline for additional lipid measures are shown in Table 2.

\section{SAFETY}

Adverse events were reported in $76 \%$ of the patients who received inclisiran and in $76 \%$ of the patients who received placebo (Table 3). Most of these events $(95 \%)$ were mild or moderate in severity (grade 1 or 2). The incidence of serious adverse events was $11 \%$ among patients who received inclisiran and $8 \%$ among patients who received placebo. Two patients discontinued participation in the trial because of adverse events: one because of a herpes zoster infection (placebo group) and the other because of influenza or nasopharyngitis (two-dose 100-mg inclisiran group). The most common adverse events (occurring in $>2 \%$ of patients) were myalgia, headache, fatigue, nasopharyngitis, back pain, hypertension, diarrhea, and dizziness, and the incidences of these events did not differ significantly between groups receiving inclisiran and those receiving placebo. Injection-site reactions occurred in $4 \%$ of the patients who received a single dose and in $7 \%$ of the patients who received two doses (after one or both doses) of inclisiran (combined rate, 5\%); injection-site reactions occurred in no patients assigned to placebo (Table 3).

Two patients had increased levels of hepatic aspartate aminotransferase ( $>3$ times the upper limit of the normal range), one in the single-dose placebo group and one in the single-dose $300-\mathrm{mg}$ inclisiran group; the patient in the 300 -mg incli- 


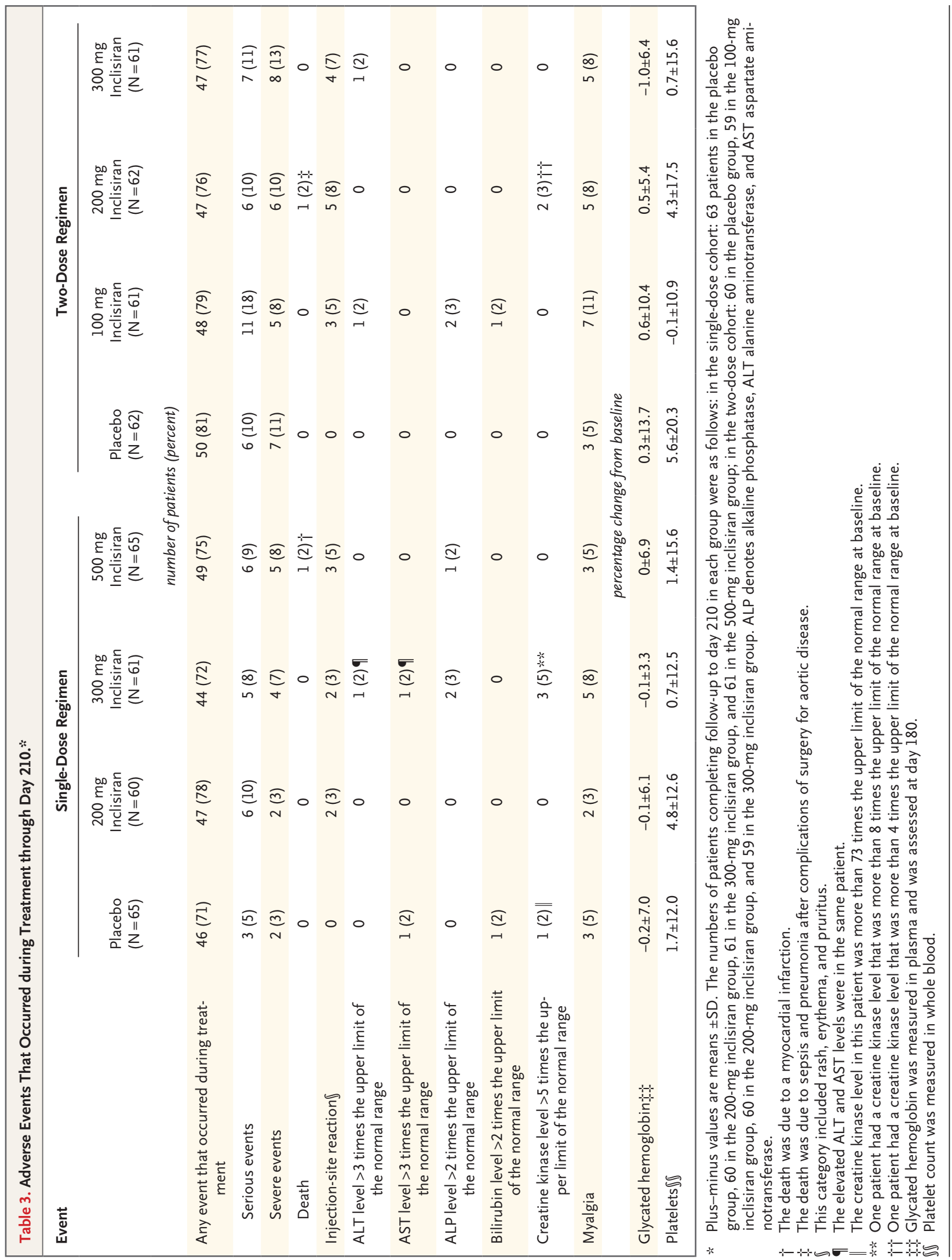


siran group also had elevations in hepatic alanine aminotransferase levels. Two additional patients (one in the two-dose 100-mg inclisiran group and one in the two-dose 300-mg inclisiran group) also had elevations in alanine aminotransferase levels. All aminotransferase elevations were transient. There were no increases in bilirubin levels that occurred in association with inclisiran or placebo among patients who had normal levels of bilirubin at baseline, and no case met the definition of Hy's law, which states that a patient is at high risk for a fatal drug-induced liver injury if given a medication that causes hepatocellular injury (not cholestatic injury) with jaundice. One patient was positive for antidrug antibodies before the first injection; no other cases of antidrug antibody were reported.

Two deaths occurred late in the trial. The first occurred in a patient who had been randomly assigned to the single-dose 500 -mg inclisiran group and who had long-standing vasculopathy and frequent angina. He had a witnessed cardiac arrest and died at 104 days. The second death occurred in a man in the two-dose 200-mg inclisiran group who had a thoracic aortic aneurysm repaired percutaneously after study entry and in whom a fistula and sepsis subsequently developed; he died 198 days into the trial.

\section{DISCUSSION}

Among patients who had a high risk of cardiovascular disease and had high LDL cholesterol levels despite receiving the maximum possible dose of statin therapy, inclisiran treatment resulted in a significantly greater reduction in LDL cholesterol levels at 180 days than did placebo. In a finding consistent with this result, as well as with the design of the drug, levels of PCSK9 were also significantly reduced at 180 days among patients who received inclisiran.

The greatest reduction (52.6\%) in LDL cholesterol levels was observed in association with the two-dose 300-mg regimen of inclisiran, a reduction that is in a range similar to that achieved with monoclonal antibodies designed to target PCSK9. Changes in other lipid measures in patients who received inclisiran were also broadly concordant, in both direction and magnitude, with results reported among patients who received anti-PCSK9 monoclonal antibodies. In the twodose 300-mg inclisiran group, every patient had a reduction in LDL cholesterol level, with a mean reduction of $64.2 \mathrm{mg}$ per deciliter $(1.66 \mathrm{mmol}$ per liter) at 180 days. At day 240, PCSK9 levels were $56.1 \%$ lower and LDL cholesterol levels $47.2 \%$ lower than at baseline, with a mean absolute reduction in LDL cholesterol level of $58.9 \mathrm{mg}$ per deciliter (1.52 mmol per liter). Taken together, these data suggest that inhibiting the translation of PCSK9 mRNA in the liver represents an alternative to targeting circulating PCSK9 and would almost certainly involve a lower injection burden.

Maintaining consistent and effective reductions in LDL cholesterol levels in the long term through the use of statins is, in part, hindered by adherence. Variability in adherence can result in considerable variability in the long-term, time-averaged reduction in LDL cholesterol levels achieved. A body of evidence now suggests that lower longterm variability in causal biologic factors is associated with a lower risk of cardiovascular disease. ${ }^{13-15}$ Hence, approaches that not only lower LDL cholesterol levels safely but also can maintain reductions consistently over time, when applied either in lieu of or simultaneously with statin therapy, are being sought.

The usefulness of targeting circulating PCSK9 as a means of reducing LDL cholesterol levels and cardiovascular risk is demonstrated by the Further Cardiovascular Outcomes Research with PCSK9 Inhibition in Subjects with Elevated Risk (FOURIER) trial, ${ }^{16}$ in which evolocumab was shown to have efficacy in reducing major cardiovascular events (primary end point) and the composite of cardiovascular death, myocardial infarction, or stroke (secondary end point) in patients with established cardiovascular disease and elevated LDL cholesterol levels. However, this approach comes with a minimal injection burden of a 3.5-ml injection 12 times per year, or a maximal burden of $261-\mathrm{ml}$ injections per year, and it still remains unknown whether the LDL cholesterollowering effects achieved with evolocumab and inclisiran will translate into similar reductions in cardiovascular events.

During 210 days of exposure to inclisiran, the rates of serious adverse events were $11 \%$ among patients receiving the drug and $8 \%$ among patients receiving placebo. Injection-site reactions were uncommon and occurred in $4 \%$ of the patients who received one dose of inclisiran and $7 \%$ of the patients who received two doses of inclisiran; these rates are similar to the rates 
observed with monoclonal antibodies to PCSK9 $9^{17,18}$ and are in contrast to the rates of 76 to $84 \%$ reported with first-generation antisense oligonucleotides for LDL cholesterol reduction..$^{19,20}$ Symptoms of immune activation, which is often a concern with therapies targeting RNA, were rare in association with inclisiran; there were few instances of flulike symptoms and no observed elevations in C-reactive protein. We observed no effects on platelet levels among patients receiving inclisiran, in contrast to recent reports from studies of antisense oligonucleotides and other siRNA molecules. ${ }^{21}$ We observed transient elevations in hepatic enzyme levels in three patients receiving inclisiran.
A trial of this size and duration cannot rule out infrequent serious side effects or side effects that might emerge after a longer period of observation. Hence, patients in this trial will be offered the chance to enter an open-label longterm safety study, although larger studies are clearly also warranted. The large majority of patients in the ORION-1 trial were of European descent: further study is warranted to determine whether inclisiran has the same effects in persons of non-European ancestry.

Supported by the Medicines Company.

Disclosure forms provided by the authors are available with the full text of this article at NEJM.org.

\section{APPENDIX}

The authors' affiliations are as follows: the Imperial Centre for Cardiovascular Disease Prevention, Department of Primary Care and Public Health, Imperial College London, London (K.K.R.), and Knowle House Surgery, Plymouth (T.H.) - both in the United Kingdom; the Department of Cardiology, Charité-Universitätsmedizin Berlin, Berlin Institute of Health and German Center for Cardiovascular Research, Berlin (U.L.), and University Heart Center Hamburg, Department of General and Interventional Cardiology, Hamburg (M.K.) - all in Germany; the Division of Endocrinology and Metabolism, Li Ka Shing Knowledge Institute and Keenan Research Centre for Biomedical Science, St. Michael's Hospital, University of Toronto, Toronto (L.A.L.); the Medicines Company, Parsippany, NJ (D.K., P.W.); Institut de Recherches Cliniques de Montréal, University of Montreal, Montreal (R.D.); the Department of Cardiology and Interventional Cardiology, VieCuri Medical Center for Northern Limburg, Venlo (R.P.T.T.), University Medical Center, Utrecht (F.L.J.V.), and the Department of Vascular Medicine, Academic Medical Center-University of Amsterdam, Amsterdam (J.J.P.K.) - all in the Netherlands; the Metabolic and Atherosclerosis Research Center, Medpace, Cincinnati (T.T.); and the Department of Cardiology, Mayo Clinic, Rochester, MN (R.S.W.).

REFERENCES

1. Goldstein JL, Brown MS. A century of cholesterol and coronaries: from plaques to genes to statins. Cell 2015;161:161-72.

2. Ridker PM, Mora S, Rose L. Percent reduction in LDL cholesterol following high-intensity statin therapy: potential implications for guidelines and for the prescription of emerging lipid-lowering agents. Eur Heart J 2016;37:1373-9.

3. Zhang H, Plutzky J, Skentzos S, et al. Discontinuation of statins in routine care settings: a cohort study. Ann Intern Med 2013;158:526-34.

4. Cohen JC, Boerwinkle E, Mosley TH Jr, Hobbs HH. Sequence variations in PCSK9, low LDL, and protection against coronary heart disease. N Engl J Med 2006;354: 1264-72.

5. Watts GF, Chan DC, Dent R, et al. Factorial effects of evolocumab and atorvastatin on lipoprotein metabolism. Circulation 2017;135:338-51.

6. Reyes-Soffer G, Pavlyha M, Ngai C, et al. Effects of PCSK9 inhibition with alirocumab on lipoprotein metabolism in healthy humans. Circulation 2017;135: 352-62.

7. Wittrup A, Lieberman J. Knocking down disease: a progress report on siRNA therapeutics. Nat Rev Genet 2015;16:54352.
8. Mello CC, Conte D Jr. Revealing the world of RNA interference. Nature 2004; 431:338-42.

9. Carthew RW, Sontheimer EJ. Origins and mechanisms of miRNAs and siRNAs. Cell 2009;136:642-55.

10. Bernards R. Exploring the uses of RNAi - gene knockdown and the Nobel Prize. N Engl J Med 2006;355:2391-3.

11. Fitzgerald K, White S, Borodovsky A, et al. A highly durable RNAi therapeutic inhibitor of PCSK9. N Engl J Med 2017; 376:41-51.

12. Nakamura M, Kayamori $Y$, Iso $H$, et al. LDL cholesterol performance of beta quantification reference measurement procedure. Clin Chim Acta 2014;431:288-93. 13. Rothwell PM, Howard SC, Dolan E, et al. Prognostic significance of visit-tovisit variability, maximum systolic blood pressure, and episodic hypertension. Lancet 2010;375:895-905.

14. Bangalore S, Breazna A, DeMicco DA, Wun CC, Messerli FH. Visit-to-visit lowdensity lipoprotein cholesterol variability and risk of cardiovascular outcomes: insights from the TNT trial. J Am Coll Cardiol 2015;65:1539-48.

15. Bangalore S, Fayyad R, Messerli FH, et al. Relation of variability of low-density lipoprotein cholesterol and blood pressure to events in patients with previous myocardial infarction from the IDEAL Trial. Am J Cardiol 2017;119:379-87.

16. Sabatine MS, Giugliano RP, Keech AC, et al. Evolocumab and clinical outcomes in patients with cardiovascular disease. N Engl J Med. DOI: 10.1056/NEJMoa1615664.

17. Sabatine MS, Giugliano RP, Wiviott SD, et al. Efficacy and safety of evolocumab in reducing lipids and cardiovascular events. N Engl J Med 2015;372:1500-9.

18. Robinson JG, Farnier M, Krempf M, et al. Efficacy and safety of alirocumab in reducing lipids and cardiovascular events. N Engl J Med 2015;372:1489-99.

19. KYNAMRO (mipomersen sodium) prescribing information. Cambridge, MA: Genzyme (http://medlibrary.org/lib/rx/meds/ kynamro-1/).

20. Raal FJ, Santos RD, Blom DJ, et al. Mipomersen, an apolipoprotein B synthesis inhibitor, for lowering of LDL cholesterol concentrations in patients with homozygous familial hypercholesterolaemia: a randomised, double-blind, placebo-controlled trial. Lancet 2010;375:998-1006.

21. Chi X, Gatti P, Papoian T. Safety of antisense oligonucleotide and siRNA-based therapeutics. Drug Discov Today 2017 January 31 (Epub ahead of print).

Copyright (c) 2017 Massachusetts Medical Society. 